\title{
SPOLM2O19
}

XIX SIMPÓSIO DE PESQUISA OPERACIONAL E LOGISTICA DA MARINHA RIO DE JANEIRO, RJ, BRASIL - 06 A 08 DE NOVEMBRO DE 2019

\section{A utilização do Solver na separação de pedidos de material de uma Organização Militar Fornecedora (OMF)}

\author{
Capitão-Tenente (QC-IM) Raphael da Silva Braga \\ Centro de Instrução e Adestramento Almirante Newton Braga/Marinha do Brasil \\ Base de Abastecimento da Marinha - Av. Brasil, 10500 - Olaria, Rio de Janeiro - RJ \\ raphael.braga@marinha.mil.br \\ Capitão de Mar-e-Guerra (RM1-IM) Hilton de Araújo Lopes \\ Centro de Instrução e Adestramento Almirante Newton Braga/Marinha do Brasil \\ Base de Abastecimento da Marinha - Av. Brasil, 10500 - Olaria, Rio de Janeiro - RJ \\ hlopes1970@hotmail.com \\ Capitão de Corveta (IM) Tiago Fernandes Lima \\ Centro de Instrução e Adestramento Almirante Newton Braga/Marinha do Brasil \\ Base de Abastecimento da Marinha - Av. Brasil, 10500 - Olaria, Rio de Janeiro - RJ \\ fernandes.lima@marinha.mil.br
}

\section{RESUMO}

Este artigo buscou investigar de que forma a separação de pedidos de material, em uma Organização Militar Fornecedora, pode ser otimizada. Para isso, realizou-se um estudo de caso em uma Organização Militar, com a utilização do Solver, um suplemento do Excel capaz de resolver modelos matemáticos, como o Problema do Caixeiro Viajante (PCV). O referido software foi utilizado como uma ferramenta de apoio para aplicação do modelo de roteirização. Com base nos dados obtidos, foi possível concluir que o emprego do Solver foi capaz de otimizar a separação de pedidos na Organização Militar (OM).

Palavra-chave: Organização Militar Fornecedora. Separação de Pedidos. Problema do Caixeiro Viajante. Método Clarke-Wright. Solver.

\begin{abstract}
This paper sought to investigate how order picking in a Supplying Military Organization can be optimized. For this, a case study was performed in a Military Organization, using Solver, an Excel supplement capable of solving mathematical models, such as the Traveling Salesman Problem (TSP). This software was used as a support tool for applying the scripting model. Based on the data obtained, it was possible to conclude that the use of Solver was able to optimize order picking in the Military Organization (OM).

Keywords: Supplier Military Organization. Order Separation. Traveling Salesman Problem. Clarke-Wright Method. Solver

Como Citar: BRAGA, R. S.; LOPES, H. A.; LIMA, T. F.; A utilização do Solver na separação de pedidos de material de uma Organização Militar Fornecedora (OMF). In: SIMPÓSIO DE PESQUISA OPERACIONAL E LOGÍSTICA DA MARINHA, 19., 2019, Rio de Janeiro, RJ. Anais [...]. Rio de Janeiro: Centro de Análises de Sistemas Navais, 2019.
\end{abstract}

\section{INTRODUÇÃO}


Os Órgãos Públicos devem buscar a eficiência, um princípio constitucional da Administração Pública, conforme previsto no caput do art. 37 da Constituição Federal (1988), e que deve estar incorporada às rotinas de cada membro da força de trabalho da Marinha do Brasil (MB) como um valor arraigado à cultura naval.

Nesse sentido, dentre as atividades necessárias ao emprego da Força, o Sistema de Abastecimento da Marinha (SAbM) objetiva promover, manter e controlar o provimento do material necessário à manutenção das Organizações Militares (OM) em condição de plena eficiência. Para isso, ele é constituído de órgãos, processos e recursos que são interligados e interdependentes.

Incluído na estrutura do $\mathrm{SAbM}$, a OM objeto de estudo, denominada $O M X$, a fim de não se expor a organização, tem como tarefas armazenar e fornecer às outras OM o material do Símbolo de Jurisdição (SJ) sob sua responsabilidade. Dessa forma, para cumprir sua missão, aquela OM executa dentro da área de armazenagem, dentre outros, o processo de separação de pedidos (recolhimento dos itens na prateleira) ao coletar materiais nas quantidades solicitadas pelos clientes. Entretanto, para a realização da separação de pedidos a OM X utiliza um processo empírico.

Essa separação de pedidos é um processo essencial à remessa de diversos materiais para as OM localizadas em todo o território nacional e demanda recursos humanos, materiais e financeiros. Saber utilizar os recursos disponíveis de forma racional indispensável para as organizações, uma vez que a redução dos custos e o aumento da qualidade do nível de serviço se tornaram elementos decisivos para a consolidação de uma empresa no mercado (JUNIOR, 2013).

Ademais, a $O M X$, alinhada às exigências da Administração Pública contemporânea e conforme estabelecido em Brasil (2018), possui, como visão de futuro ser reconhecida como uma Organização Militar Fornecedora (OMF) moderna e tecnologicamente atualizada que contribua para o aprimoramento do SAbM e seja reconhecida pelo padrão de excelência no atendimento às $\mathrm{OM}$ clientes.

Sendo assim, com base nas exigências de eficiência na Administração Pública e no previsto como visão de futuro da $O M X$, bem como na importância do processo de separação de pedidos, formula-se o problema de pesquisa: o Solver pode ser utilizado como ferramenta eficaz para a otimização da separação de pedidos da $O M X$ ?

Para responder a esta pergunta, definiu-se como objetivo principal: verificar se o Solver é uma ferramenta útil para a separação de pedidos na $O M X$. Além disso, foram estabelecidos os seguintes objetivos específicos: analisar o processo de recolhimento de pedidos na $O M X$ e comparar o deslocamento em metros realizado sem o Solver com os resultados da utilização dele no recolhimento de materiais nas prateleiras.

Cabe ressaltar que a OM objeto deste estudo foi escolhida por ser um dos Depósitos do Complexo Naval de Abastecimento com maior demanda média mensal de Requisições de Material (RM). Ademais, essa OM conta com um dos maiores efetivos de pessoal envolvido na atividade de separação de pedidos e possui a maior diversidade de SJ sob responsabilidade de uma OMF.

Então, a realização deste trabalho justifica-se diante do exposto acima e considerando a inexistência de pesquisas que analisam especificamente o processo de separação de pedidos nas OMF.

Outrossim, este estudo busca ser capaz de fornecer informações relevantes para apoiar decisões gerenciais e visa contribuir para a busca da excelência no cumprimento da missão da $\mathrm{MB}$, enquanto parte da Administração Pública. Até porque, ainda não está disponível na $O M X$ a utilização do Warehouse Management System (WBS). Para Gasnier e Banzato (2001), um WMS é um sistema de gestão de armazéns, que otimiza todas as 
atividades operacionais (fluxo de materiais) e administrativas (fluxo de informações) dentro do processo de armazenagem.

Quanto à organização deste artigo, esta primeira parte dedica-se a uma contextualização, além da apresentação do problema da pesquisa e dos objetivos a serem perseguidos. Na segunda, será apresentada a base teórica que reveste este estudo. Na terceira seção será exposta a metodologia da qual o autor se serviu para chegar a uma conclusão. $\mathrm{Na}$ quarta seção os dados obtidos serão submetidos à análise. Por fim, as conclusões e sugestões para pesquisas futuras serão apresentadas na quinta seção.

\section{REVISÃO BIBLIOGRÁFICA}

\subsection{O Sistema de Abastecimento da Marinha (SAbM)}

Conforme Brasil (2009), o SAbM tem como objetivo promover, manter e controlar o provimento do material necessário para que as OM atuem em condição de plena eficiência. Para atingir seus objetivos o SAbM desenvolve atividades de dois tipos: gerenciais e técnicas.

O primeiro tipo, as técnicas, refere-se àquelas de caráter administrativo, diretamente relacionadas com a manutenção do fluxo adequado do material necessário às $\mathrm{OM}$. $\mathrm{O}$ segundo tipo de atividade diz respeito à elaboração de normas que assegurem a consecução dos padrões a serem observados e dos resultados esperados com a sua utilização.

Incluído no tipo de atividade gerencial, o fornecimento é a atividade relacionada com a entrega do material ao utilizador e é realizado pela OMF. Conforme Brasil (2016), as OMF têm a finalidade de armazenar e fornecer o material destinado às outras OM.

Um dos tipos de OMF são os Depósitos Primários:

OM de apoio, de âmbito nacional, dentro da cadeia de comando da Diretoria de Abastecimento da Marinha (DAbM), responsáveis pela distribuição do material de determinada categoria, devidamente selecionada e especificada (BRASIL, 2009, p. 8-9).

Segundo Brasil (2009), os materiais armazenados nas OMF são agrupados em função de sua natureza específica por um código alfabético denominado "Símbolo de Jurisdição" (SJ). De modo geral, a fim de que um material seja fornecido, uma OM precisa inserir sua necessidade em uma Requisição de Material (RM) no Sistema de Informações Gerenciais do Abastecimento (SINGRA), sistema esse baseado em processamento de dados e estabelecido e mantido pela DAbM. "A RM é o documento, estabelecido no âmbito do SAbM, que formaliza o pedido de material às OMF." (BRASIL, 2016, p. 2-13).

$\mathrm{Na} \mathrm{MB}$, uma das categorias de material fornecidas pelo SAbM, a partir de uma RM, é a de material comum. Esse tipo possui como características o consumo frequente, a grande popularidade, a pouca complexidade tecnológica, a aplicação na conservação, na limpeza, na arrumação, na apresentação, na segurança e no processo administrativo-burocrático das OM.

Os itens da MB que possuem SJ "E", "VG", "CG", e "G" são genericamente tratados como itens de material comum. Os de SJ "E" são, dentre outros, as tintas e lubrificantes. O de SJ "VG" são equipagens para operações aéreas. O "CG" refere-se aos itens utilizados em atividades marinheiras - "cordas de marinheiro", por exemplo - como os de "Controle de Avarias, os de Marinharia e os de Salvatagem" (BRASIL, 2009, p. A2). Por fim, os itens "G" são aqueles não contemplados nas outras classificações, como papel A4 e canetas, dentre outros.

\subsubsection{A $O M X$}


Integrante do SAbM, a $O M X$ é uma OMF com atividade relacionada ao abastecimento das $\mathrm{OM}$ da $\mathrm{MB}$ situadas em todo o território nacional, no que diz respeito ao material sob sua jurisdição, descritos no Quadro 1.

\begin{tabular}{|c|c|}
\hline \multicolumn{2}{|c|}{ Quadro 1- Materiais sob responsabilidade da OM X } \\
\hline Item & Símbolo de Jurisdição (SJ) \\
\hline Gêneros Alimentícios & MIKE (M) \\
\hline Material Comum & GOLF (G) \\
\hline Tintas e Correlatos -1 & $\mathrm{ECHO}(\mathrm{E})$ \\
\hline CAv, Marinharia e Salvatagem & CHARLIE GOLF (CG) \\
\hline Equipagens para operações aéreas & VICTOR GOLF (VG) \\
\hline Tintas e Correlatos -2 & ROMEO ECHO (RE) \\
\hline
\end{tabular}

Uma das etapas precedentes ao fornecimento dos materiais do Quadro 1 é "a separação dos pedidos contidos nas Requisições de Material liberadas pelo Centro de Controle de Inventário da Marinha (CCIM) no SINGRA, para o posterior fornecimento às OM" (BRASIL, 2018, p. 13). Em relação aos itens de material comum de SJ "CG" e "G", a $O M X$ os acondiciona em cinco armazéns diferentes, ocupando uma área de $2.205,5 \mathrm{~m}^{2}$, com cerca de três mil itens distintos.

O $O M X$ é subordinado ao CCIM. Esse Centro possui, dentre outras funções, a de controlar o inventário do material comum, mantendo o adequado equilíbrio entre as necessidades da Marinha e as disponibilidades de material nas OMF. Como consequência, é o CCIM que libera o fornecimento do material armazenado naquelas OM.

\subsection{Separação de pedidos, o Problema do Caixeiro Viajante (PCV) e a roteirização pelo método Clarke-Wright}

Ainda no contexto dos armazéns, segundo Tortola et al. (2017), a separação de pedidos em um ambiente de abastecimento de materiais define-se como a coleta do mix correto de produtos, em suas quantidades corretas da área de armazenagem para satisfazer as necessidades do consumidor. Essa atividade "[...] normalmente representa grande parte dos custos do depósito" (NETO; CARDOSO, 2014, p. 3).

Em virtude disso, para que a separação de pedidos não seja um problema para o orçamento financeiro das organizações, Trevisan et al. (2018) propõem a otimização da roteirização: o atendimento eficiente de nós de demanda geograficamente dispersos.

Cunha (2000), afirma que a roteirização realiza o sequenciamento de paradas geograficamente dispersas que devem ser efetuadas a fim de prover o atendimento a todos os locais previamente estabelecidos. Laporte (2000) complementa que normalmente esse mecanismo tem como objetivo iniciar uma ou mais rotas que tenham como ponto de partida e chegada um terminal de cargas ou depósito.

Segundo Neto e Cardoso (2014), no tocante à roteirização há o conceito do Problema do Caixeiro Viajante (PCV), esse que aborda a determinação da rota que minimiza uma distância total, a partir do conhecimento de determinadas restrições.

No PCV os pontos de origem e destino são coincidentes, de forma que a viagem não é considerada completa até o retorno do veículo ao ponto de partida. Ou seja, "o objetivo é achar a sequência na qual os pontos visitados minimizam a distância ou o tempo total de viagem" (BALLOU, 2006, p. 197).

Entretanto, como ressaltado por Ballou (2006), não é fácil encontrar o melhor roteiro para um determinado problema quando há muitos pontos ou quando uma solução rápida é necessária. Por isso, procedimentos informatizados especiais têm sido desenvolvidos no 
sentido de resolver com rapidez o problema espacialmente representado e de produzir resultados que chegam perto do ótimo.

Para Neto e Cardoso (2014), a roteirização de veículos pode ser considerada dentro de armazéns, os quais em geral, são formados por um conjunto de corredores de acesso e um conjunto de prateleiras, nas quais são armazenados os produtos. "As rotas que minimizam as distâncias percorridas serão definidas de acordo com as localizações e posições das prateleiras e corredores do depósito" (NETO; CARDOSO, 2014, p. 6). Por esse motivo, as localizações das prateleiras são o conhecimento base para o estudo da roteirização em depósitos.

"Com relação aos métodos de resolução do PCV, destaca-se o método Clarke-Wright de roteirização, também conhecido como método das economias" (NETO e CARDOSO, 2014, p. 6), método base para este trabalho. Isso porque, segundo Ballou (2006), o método Clarke-Wright é flexível e suficiente para resolver uma ampla coleção de restrições práticas impostas pelo PCV. Para Neto e Cardoso (2014), esse método tem sido extensivamente utilizado na solução de problemas de roteamento, devido a sua eficiência e simplicidade. Logo, "o objetivo é minimizar a distância total percorrida, respeitando todas as restrições impostas pelo problema" (NETO; CARDOSO, 2014, p. 6).

Primeiro, a lógica do método está em considerar o deslocamento de ida e de volta de um ponto qualquer (depósito) até dois outros pontos diferentes (A e B), conforme a Figura 1.

Figura 1 - Rota inicial considerada pelo método Clarke-Wright

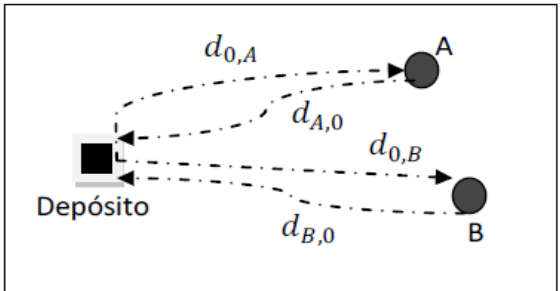

Fonte: Adaptado de Ballou (2006, p. 205).

A Figura 1 fornece a distância máxima a ser abordada no problema da roteirização. Para dar continuidade ao método Clarke-Wright, combinam-se duas paradas no mesmo roteiro a fim de tornar possível a redução da distância a ser percorrida. Para determinar quais paradas a serem combinadas num roteiro, calcula-se o total das distâncias antes e depois da combinação.

Como consequência, a distância economizada pela combinação de dois pontos (A e B) é calculada pela subtração algébrica da distância da rota. $O$ resultado é um valor economizado $\mathrm{S}_{\mathrm{A}, \mathrm{B}}=\mathrm{d}_{0, \mathrm{~A}}+\mathrm{d}_{0, \mathrm{~B}}-\mathrm{d}_{\mathrm{A}, \mathrm{B}}$, apresentado na Figura 2 .

Figura 2- Ilustração dos ganhos ao agrupar pontos de demanda A e B em uma mesma rota

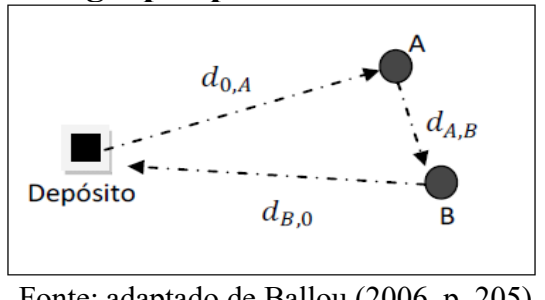

Fonte: adaptado de Ballou (2006, p. 205) 
Este cálculo é feito para todas as combinações de paradas. Como ilustrado na Figura 2, "o par de escalas com o maior valor economizado é escolhido para a combinação" (BALLOU, 2006, p. 205).

$\mathrm{O}$ processo de combinação de rotas continua. Para finalizar, o maior valor de economia identifica a parada que deveria ser levada em conta para a inclusão num roteiro. Para Hillier (2001), o objetivo é encontrar o caminho mais curto (o caminho com a distância total mínima) desde a origem até o destino.

Para Ballou (2006) o processo interativo avança até que todas as paradas sejam examinadas. Segundo Hillier (2001) o problema geral de transporte está preocupado com a distribuição de qualquer mercadoria de qualquer grupo de centros de fornecimento, chamados fontes, para qualquer grupo de centros receptores, chamados destinos, de modo a minimizar o custo total de distribuição.

Para Ballou (2006), esta abordagem não é garantia de uma solução ótima, mas, levando-se em conta a complexa natureza desse problema, aumenta as probabilidades de que se alcance uma boa solução.

\subsubsection{A Pesquisa Operacional (PO) e o Solver}

A Pesquisa Operacional (PO) pode ser usada para facilitar a análise e solução do método Clarke-Wright, relacionado ao PCV.

Para Cardoso (2011), a PO designa uma área do conhecimento que consiste no desenvolvimento de métodos científicos de sistemas complexos, com a finalidade de prever e comparar estratégias ou decisões alternativas, cujo objetivo é dar suporte à definição de políticas e à determinação de ações, com base em modelagem matemática.

Um exemplo dessa modelagem em uma Pesquisa Operacional pode ser analisado na Figura 3.

Figura 3 - Exemplo de Modelagem Matemática de uma PO

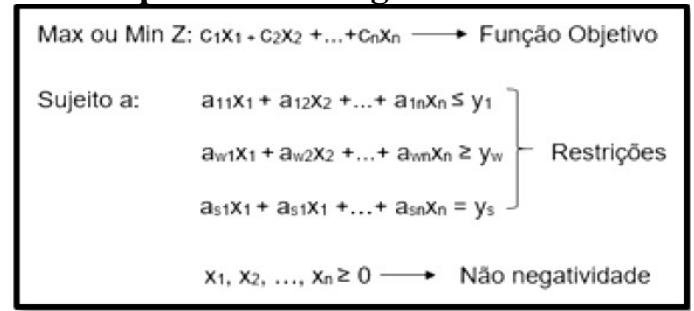

Fonte: Adaptado de Angelini et al. (2017).

A aplicação da modelagem de uma PO como a da Figura 3, com a função objetivo e algumas restrições, é presente em diversas áreas "onde se deseja aperfeiçoar a produção, diminuir a perda de matéria-prima e de transporte e onde se busca otimizar as rotas com menor tempo e custo de viagem" (ANGELINI et al., 2017, p. 2). Ou seja, "o modelo matemático obtém a melhor solução" (SOHN, 2018, p. 3) e mostra-se útil para problemas antes considerados complexos.

A função objetivo da Figura 3 serve para medir o comportamento do sistema, ou seja, o quanto será gerado de lucro ou reduzido o custo. Caso o objetivo seja maximizar o lucro, as restrições e a não negatividade garantem que essa solução esteja de acordo com as limitações técnicas impostas pelo sistema.

Segundo Marins (2011), estes métodos matemáticos encontram-se em crescente evolução e, por isso, foram desenvolvidos diversos softwares que auxiliam a resolução dos modelos matemáticos da PO. Dessa forma, ainda segundo Marins (2011), torna-se viável e eficiente a solução de problemas complexos a partir da utilização do Solver, por exemplo, suplemento do software Microsoft Excel. 
Com base em consulta no sítio eletrônico da Microsoft (2018), com o Solver pode-se encontrar um valor ideal, máximo ou mínimo, para uma fórmula em uma célula objetivo conforme as restrições, ou limites, sobre os valores de outras células de uma planilha.

Ainda segundo o sítio eletrônico, trabalhando com um grupo de células, chamadas variáveis de decisão ou simplesmente de células variáveis, que participam do cálculo das fórmulas nas células de objetivo e de restrição, o Solver ajusta os valores nas células variáveis de decisão para satisfazer aos limites sobre células de restrição e produzir o resultado desejado para a célula objetiva. Dessa forma, o Solver, uma ferramenta da PO, é capaz de auxiliar a resolução do PCV, já que o citado suplemento é capaz de resolver as restrições de uma modelagem matemática.

Lima et al (2018) explicam que a utilização de métodos quantitativos no apoio à tomada de decisões em cenários complexos ajuda a evitar o emprego de processos pouco criteriosos, já que quando utilizado algum tipo de estratégia matemática para a sustentação da resolução de um problema, obtém-se maiores racionalidade e objetividade. Justifica-se, dessa forma, a utilização da PO nesta pesquisa.

\section{METODOLOGIA}

\subsection{Tipo de pesquisa}

Segundo Prodanov e Freitas (2013), do ponto de vista de sua natureza, este trabalho é uma pesquisa aplicada, pois tem como objetivo gerar conhecimentos para aplicação prática, dirigidos à solução de problemas específicos.

Em relação à abordagem do problema, ainda segundo Prodanov e Freitas (2013), em um primeiro momento este trabalho é qualitativo porque o ambiente natural foi a fonte direta para coleta de dados, interpretação de fenômenos e atribuição de significados. Posteriormente, a abordagem foi quantitativa, pois se utilizou de recursos e técnicas de estatística ao procurar traduzir em números os conhecimentos gerados pelo pesquisador para, em seguida, formular as possíveis conclusões.

Quanto aos meios, seguindo a classificação de Vergara (2014), trata-se de um estudo de caso. A autora menciona que este método de pesquisa pode ser limitado a uma ou a poucas unidades e que pode ser, ou não, realizado em campo. Nesse sentido, Yin (2010) complementa que o estudo de caso é utilizado, dentre outras situações, para o conhecimento dos fenômenos individuais e grupais. Ele entende que os estudos de caso podem ser exploratórios e explicativos.

As autoras Markoni e Lakatos (2010) acrescentam que os estudos exploratórios e explicativos combinados têm o objetivo de descrever um fenômeno, por exemplo, um estudo de caso no qual são realizadas análises empíricas e teóricas.

Além disso, quanto aos tipos de instrumentos utilizados, segundo Prodanov e Freitas (2013), ela é bibliográfica, pois foi elaborada a partir de material já publicado, constituído principalmente de: livros, revistas, publicações em periódicos e artigos científicos. Também, segundo Gil (2008), quanto aos instrumentos, procedeu-se a uma pesquisa documental ao analisar materiais que ainda não receberam um tratamento analítico, como os documentos normativos da MB.

\subsection{Coleta de dados}

Os dados foram coletados com base em uma amostra estabelecida por meio de seleção racional, essa que, segundo Prodanov e Freitas (2013), tem como resultado um tipo de amostragem não probabilística e consiste em selecionar um subgrupo da população com base nas informações disponíveis, apresentando resultados com validade para um contexto específico. 
Para tal, foi realizada uma observação não participante, aquela que, segundo Prodanov e Freitas (2013), o pesquisador toma contato com a comunidade, o grupo ou a realidade estudada, mas sem integrar-se a ela, a fim de coletar dados.

O objeto de estudo foi a separação de pedidos dos materiais comuns dos SJ "G" e “CG", encontrados nos armazéns $\mathrm{n}^{\mathrm{os}} 1,2,3,4$ e 6 do $O M X$. Os dois tipos foram escolhidos porque a totalidade de seus itens encontra-se acondicionada em prateleiras, o que favoreceu a realização desta pesquisa.

O $O M X$ foi escolhido por ser um dos Depósitos do Complexo Naval de Abastecimento com maior número mensal de Requisições de Material (RM) por OM clientes, por contar com um dos maiores efetivos de pessoal envolvido na atividade de separação de pedidos (37 militares, 5 servidores civis e 36 estivadores vinculados por contrato licitatório), e por possuir a maior diversidade de SJ sob responsabilidade de uma OMF.

Com relação aos estivadores verificou-se que são vinculados por contrato a uma empresa especializada na prestação de serviço de movimentação e armazenagem de carga geral. Esse contrato custa anualmente cerca de $\mathrm{R} \$ 2$ milhões a $O M X$.

Para a coleta dos dados, foram realizadas visitas a $O M X$ entre julho e outubro de 2018. Dentre outras atividades, acompanhou-se o processo de separação dos pedidos contidos nas RM. Além disso, foram conhecidas as localizações dos materiais de SJ "G" e "CG", como também os percursos passíveis de roteirização a partir da separação de pedidos para uma OM cliente.

Também, foram extraídas do SINGRA 72 Capas de Lote - documento que agrega um conjunto de RM de uma OM. Esses 72 documentos representam 306 RM, de OM diversas, com a demanda de itens do SJ "G" e "CG" relacionada ao período de $1^{\circ}$ de janeiro a 31 de agosto de 2018. Considerando a média mensal de RM atendidas pela $O M X$ por mês, a amostra representa $49 \%$.

A fim de simplificar a análise e o tratamento dos dados, os materiais comuns dos SJ selecionados foram agrupados em 36 localizações, distribuídas pelos cinco armazéns, conforme representado na Figura 4.

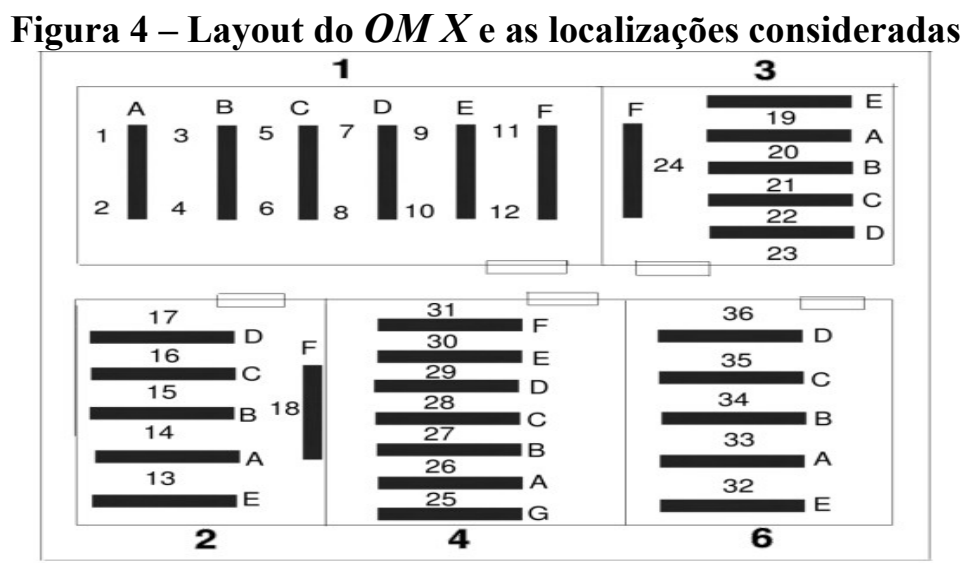

Fonte: Elaborado pelo autor a partir dos dados coletados.

Essas localizações representam o ponto no qual o separador de materiais irá se posicionar para realizar sua função.

Além disso, foi realizada a medição de distâncias em metros entre cada uma das 36 localizações entre si e entre elas e o ponto de saída de material da $O M X$.

\subsection{Tratamento de dados e software utilizado}


A lógica do PCV foi aplicada no processo de separação de pedidos da $O M X$ e a sua resolução foi obtida por meio do método Clarke-Wright, com apoio do Solver.

O objetivo do tratamento dos dados foi verificar se o processo de separação de pedidos realizado na $O M X$ poderia apresentar um deslocamento total menor em metros ao ser comparado com o resultado que um software apresentaria para as mesmas condições.

Para apoio ao tratamento dos dados levantados foi utilizada a ferramenta Solver, da Microsoft Excel. O sistema proposto tem como entrada de dados a localização de cada material coletado de uma RM e a posição do ponto de saída do depósito.

Com tais dados, o sistema apresenta como saída uma lista sequenciada de coletas com o resultado em metros que melhor satisfaz um deslocamento, de acordo com as restrições impostas.

Na execução deste trabalho, como resultado da aplicação do Solver, foi gerada a coluna "Loc" em uma planilha do Excel, uma das três colunas de células variáveis, conforme a situação hipotética apresentada na Tabela 1.

Tabela 1 - Células Variáveis

\begin{tabular}{lll}
\hline Localização Visitada & Loc & $\begin{array}{l}\text { Distância } \\
(\mathrm{m})\end{array}$ \\
\hline 1 & 1 & 66,25 \\
2 & 2 & 10 \\
3 & 2 & 0 \\
5 & 3 & 12,5 \\
6 & 3 & 0 \\
7 & 4 & 10 \\
8 & 5 & 15 \\
9 & 6 & 10 \\
2 & 6 & 0 \\
3 & 6 & 0 \\
9 & 7 & 15 \\
8 & 7 & 0 \\
6 & 8 & 10 \\
12 & 8 & 0 \\
2 & 9 & 12,5 \\
6 & 9 & 0 \\
7 & 12 & 15 \\
12 & 12 & 0 \\
15 & 15 & 37,5 \\
17 & 17 & 20 \\
\hline TOTAL & & 233,75 \\
\hline Fote & &
\end{tabular}

Fonte: Elaborado pelo autor.

A primeira coluna da Tabela 1 apresenta quais das 36 localizações foram visitadas para recolhimento de um material e em qual ordem foi realizado esse processo. Na segunda, chamada "Loc", a sequência de localizações sugerida pelo Solver para uma RM. Na terceira coluna, as distâncias reais em metros entre as localizações, com base na sequência da coluna "Loc".

Em relação à coluna "Distância", da Tabela 1, o valor de 66,25 representa o deslocamento em metros entre a última posição visitada e o ponto de expedição. A distância entre as "Loc" 1 e 2 é igual a 10 metros. A distância entre a "Loc" 2 e ela mesma é igual a 
zero. Entre a 2 e a 3 é igual a 12,5 metros. Dessa forma, continua-se o processo até a distância entre as "Loc" 15 e 17, de 20 metros. O total em metros da distância percorrida nesse roteiro sugerido pelo Solver será de 233,75 metros.

Ressalta-se que a coluna "Distância" importa valores dos deslocamentos reais entre as 36 localizações e entre elas e o ponto de saída do depósito, registradas em outra planilha do Excel, constante da Figura 5.

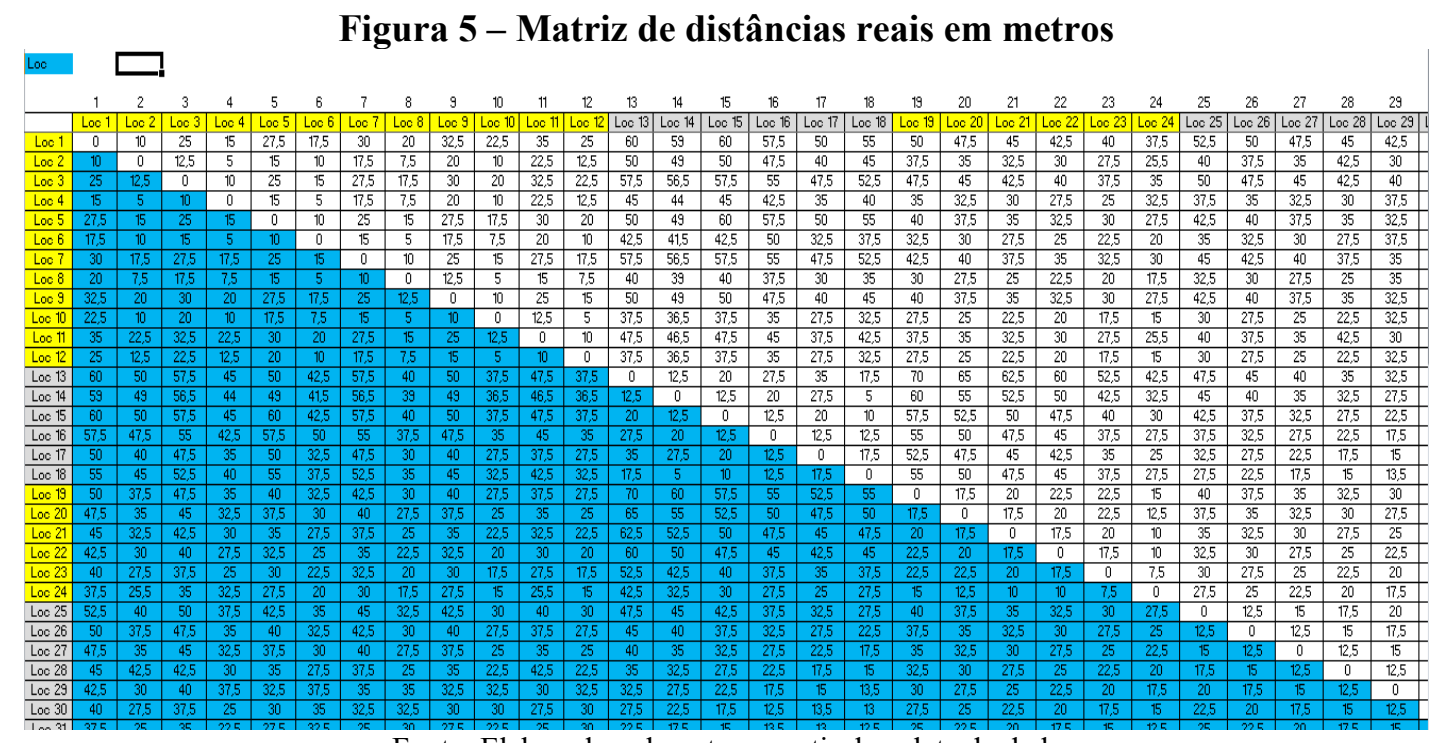

Fonte: Elaborado pelo autor a partir da coleta de dados.

Para dar continuidade ao trabalho, na mesma planilha constante da Tabela 1, selecionou-se a opção Solver do Excel. Com isso, o software apresenta a caixa de diálogo conforme a Figura 6.

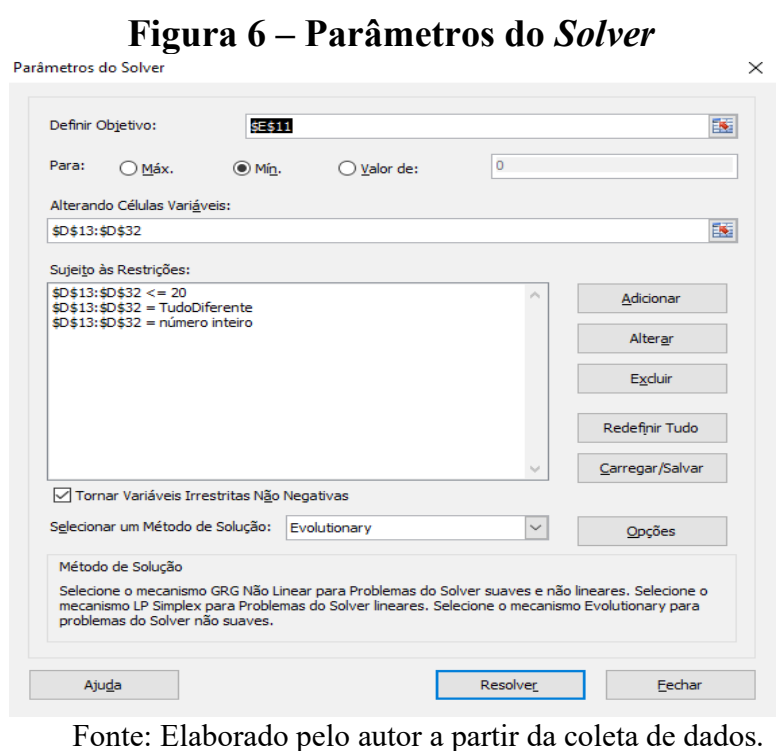

Depois, foi inserida na caixa "Definir Objetivo", como exemplo da Figura 6, a referência de célula na qual o software apresentou o resultado solicitado. Em seguida, na opção "Para", da mesma Figura, foi selecionada a opção "Mín", já que se desejou o menor valor possível de deslocamento em metros para a célula objetivo. 
Na caixa "Alterando Células Variáveis", também da Figura 6, foram selecionadas as células da primeira coluna da Tabela 1. Essas células podem ser alteradas a partir das restrições impostas na opção "Sujeito às restrições" daquela Figura. Essas restrições orientam o resultado das células "Loc", que representam o roteiro ideal sugerido pelo sistema, no sentido de que esse roteiro não apresente localizações com valores fracionados ou localizações que não foram visitadas na realidade.

Adicionalmente, para encontrar a solução ideal o sistema usa o método Evolutionary, conforme a Figura 6, esse que utiliza a lógica de buscar a solução ótima, cortando e ramificando resultados que não correspondam às restrições propostas.

Por fim, ao selecionar a opção "Resolver", constante da Figura 6, o sistema servirá como facilitador para a obtenção do cálculo das iterações e apresentará, na coluna "Loc" da Tabela 1, o roteiro que apresenta o menor deslocamento na execução do processo de separação de pedidos de uma RM qualquer.

A sequência de rota considerada real e comparada com o software leva em conta a ordem na qual as RM aparecem em uma Capa de Lote, desde a primeira até a que aparece por último. Foi considerada essa ordenação porque, por meio das observações no campo de estudo, notou-se que a separação de materiais ocorre, geralmente, dessa maneira.

\subsection{Limitações da pesquisa}

Por outro lado, não foi considerada a disponibilidade dos materiais e ferramentas necessárias para execução da atividade de separação de pedidos, assim como a capacidade exata de carregamento de material pela empilhadeira ou pelo responsável.

Além disso, como o foco é o processo de separação de pedidos, não são considerados erros em processos anteriores. Por esse motivo, os resultados apresentados pelo Excel não identificam se um material está ou não em uma localização correspondente à do sistema SINGRA. O Solver indicará o menor deslocamento em metros independentemente de qual item está na localização analisada.

Adicionalmente, considerando as 36 localizações deste trabalho, foram analisadas as Capas de Lote que continham mais de duas RM com materiais em localizações diferentes. Tal fato se deu porque o software considera que o deslocamento em metros de um ponto A até o mesmo ponto A é igual a zero, por exemplo. Nesse caso, o Solver analisaria apenas o deslocamento em metros entre a localização e o ponto de expedição.

Logo, uma Capa de Lote com itens apenas de uma mesma localização não geraria deslocamentos mínimos consideráveis relevantes para esta pesquisa. Por consequência, das 72 Capas de Lote obtidas, que totalizaram $900 \mathrm{RM}$, foram consideradas 25 delas para este estudo, 260 RM analisadas em grupos de 20, totalizando 13 resultados.

\section{RESULTADOS E DISCUSSÃO}

\subsection{Análise do processo de separação de pedidos}

A partir da pesquisa de campo, observou-se que o processo de separação de pedidos de materiais na $O M X$ é empírico e não se apoia em nenhuma atividade automatizada. Além disso, foi possível estabelecer qual a distância percorrida pelos responsáveis em separar pedidos, a fim de comparar esses resultados com um outro apresentado pelo Solver.

Dessa forma, observou-se que o processo se inicia a partir da inserção de uma RM no SINGRA pela OM cliente. Essa solicitação, após liberada pelo CCIM, passa a contar naquele sistema como "requisição em atendimento".

A $O M X$, por sua vez, realiza uma análise diária de todas a RM que foram liberadas. Um militar imprime a relação de material a ser separado, gerando um documento denominado Capa de Lote, esse que reúne várias RM de uma mesma OM. 
A separação de pedidos considerada neste trabalho é de responsabilidade da Seção de Material Comum, pertencente à Divisão de Abastecimento. Os militares daquela seção, quando da separação dos pedidos, verificam se há necessidade de utilização de empilhadeira ou não. Com a Capa de Lote em mãos deslocam-se até a prateleira onde se encontra o material e realizam a separação dos itens na ordem em que eles aparecem na Capa de Lote.

Após o recolhimento do material na prateleira o separador pode recolher o material de uma só vez ou, por limitações de capacidade de carregamento, posicionar parte dos itens solicitados no ponto de saída do depósito para continuar a atividade posteriormente. Este trabalho analisou a separação no caso de o material ser recolhido de uma só vez.

As OM solicitantes, no geral, retiram o material diretamente na $O M X$ e as localizadas em outros Distritos Navais recebem seu material por meio dos Centro de Intendência regionais. Por fim, o material entregue tem seu registro de fornecimento inserido no SINGRA, o que atualiza os estoques cadastrados.

A Figura 7, feita a partir da utilização do software Bizagi, apresenta o resumo do processo de separação de pedidos no $O M X$.

Figura 7 - O processo resumido de separação de Pedidos na $O M X$

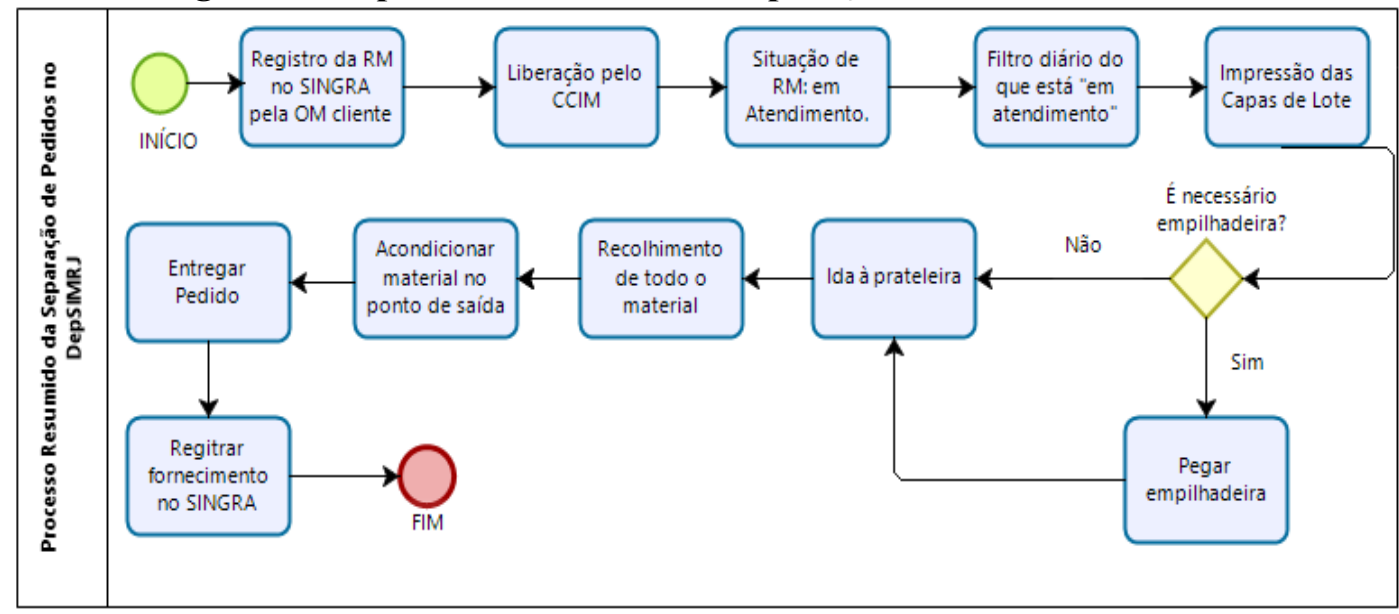

Fonte: Elaborado pelo autor com base na coleta de dados.

A partir da análise da Figura 7 percebe-se que não há uma etapa no processo de separação de materiais comuns na qual se analisa qual é o melhor roteiro a ser percorrido. Nesse contexto, considerando também as dimensões da OM, diminuir o deslocamento em metros dentro do depósito significa, dentre outros, otimizar o processo de separação de pedidos. Como essa atividade consome recursos de um depósito, torna-se necessário aplicar na $O M X$ uma sistemática capaz de melhorar aquela atividade.

Assim, ao se considerar a busca pela otimização do roteiro para a separação de pedidos no ambiente do $O M X$, pode ser aplicada a lógica do PCV, um dos primeiros problemas de roteamento apresentado na literatura e que possui como intuito, neste caso, a determinação da rota que minimizará a distância total percorrida para realizar a atividade de separação de pedidos.

A resolução do PCV no $O M X$, com o auxílio do método Clarke-Wright, pode utilizar um software como facilitador.

\subsection{Aplicação do Solver}

A partir da inserção de 25 Capas de Lote no Solver, 260 RM, observou-se que o deslocamento em metros efetivamente realizado pode ser melhorado. 
Os resultados mostraram, como apresentado no Gráfico 1, no qual o eixo y representa o deslocamento em metros e o eixo x representa cada uma das 25 Capas de Lote, que o deslocamento efetivamente realizado pelo separador é maior, na maioria das vezes, do que o deslocamento proposto pela roteirização do software.

\section{Gráfico 1 - Deslocamento Real x Software em metros}

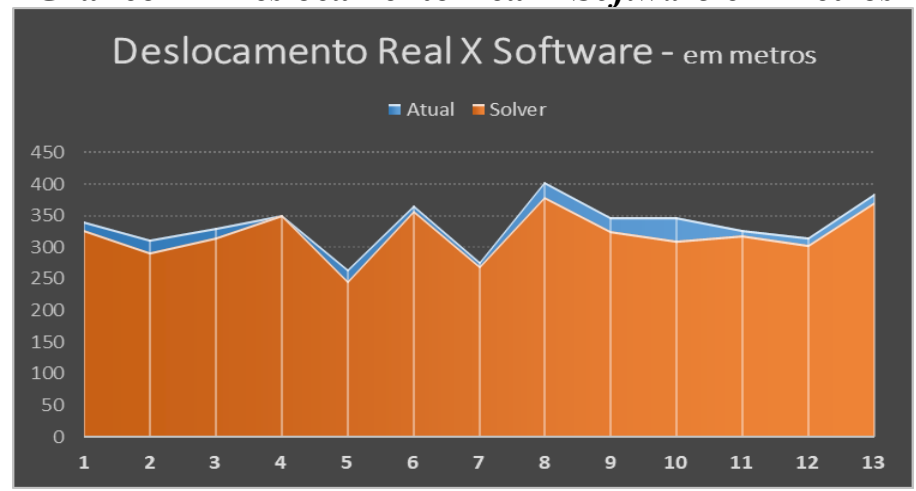

Fonte: Elaborado pelo autor a partir dos dados coletados

Ainda com base no Gráfico 1, percebe-se que o roteiro realizado pelo separador de pedidos poderia ter sido desenvolvido de forma mais eficiente para a maioria das Capas de Lote analisadas. Confirma-se o que expôs Ballou (2006) ao afirmar que o método ClarkeWright é flexível e suficiente para resolver uma ampla coleção de restrições práticas impostas pelo PCV.

Além disso, quando se compara o deslocamento total em metros necessários para separar as $260 \mathrm{RM}$, conforme o Gráfico 2, ratifica-se que a distância é menor com a utilização do software.

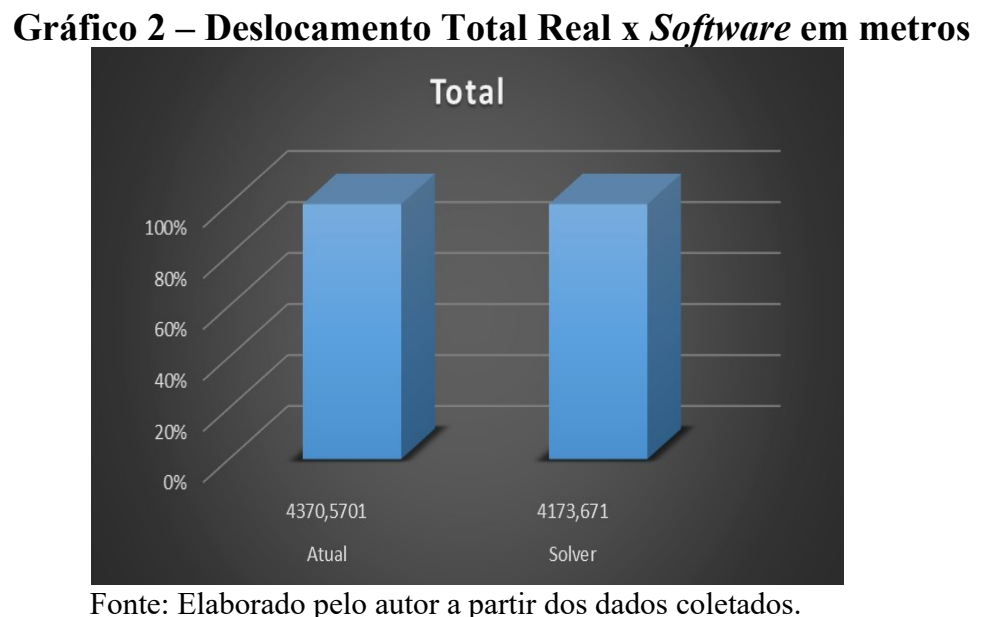

No caso, é possível perceber, a partir da análise do Gráfico 2, que o deslocamento em metros realizado pelo separador, considerando as 25 Capas de Lote, foi de 4.370,57 metros. Após a sugestão de rota realizada pelo Solver, o deslocamento para as mesmas Capas foi de 4.173,67 metros. Uma economia aproximada de 190 metros.

A Pesquisa Operacional (PO), a partir da aplicação do Solver, facilitou a análise e solução do método Clarke-Wright, relacionado ao PCV. Ou seja, o modelo matemático obteve a melhor solução, como já previsto por Sohn (2018), e mostrou-se útil para problemas antes considerados complexos.

Adicionalmente, o Gráfico 3 compara o percentual de vezes que o resultado em metros sugerido pelo software foi maior, igual ou menor do que o efetivo. 


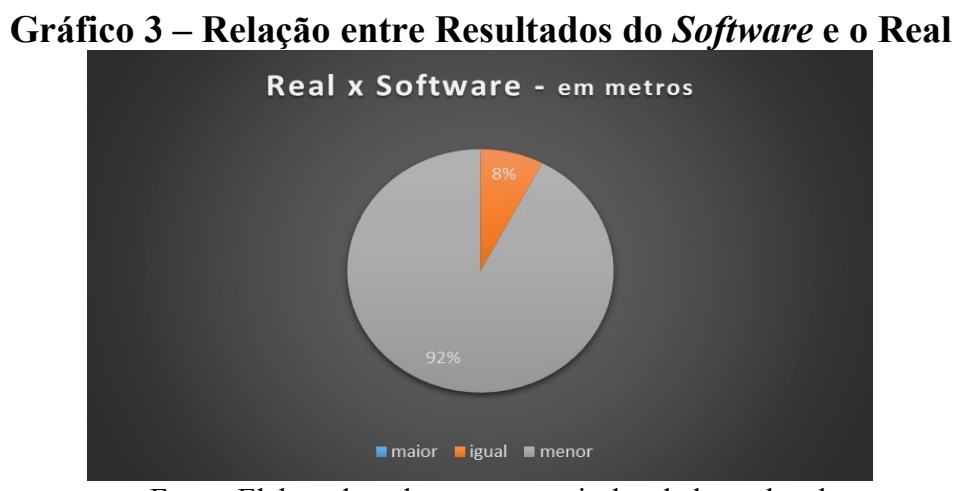

Fonte: Elaborado pelo autor a partir dos dados coletados

Com base nos dados do Gráfico 3, percebe-se que 92\% dos testes realizados pelo software apresentaram um deslocamento menor do que o executado na realidade; em $8 \%$ das vezes o descolamento realizado e o sugerido foram muito parecidos; e em nenhuma vez o software sugeriu um deslocamento maior do que o realizado na realidade. Esses resultados mostram que a aplicabilidade do software será, na maioria dos casos, vantajosa para a $O M X$.

Em que pese o Solver ter apresentado resultado total em metros próximo ao realizado atualmente, ao se ampliar a pesquisa, poderá ser observada uma economia de recursos mais significante. Dessa forma, identifica-se o software Excel, com o seu suplemento, Solver, como ferramenta capaz de otimizar a separação de pedidos na $O M X$. Assim, confirma-se o que Marins (2011) registrou: torna-se viável e eficiente a solução de problemas complexos a partir da utilização do Solver.

\section{CONCLUSÃO}

O presente estudo de caso procurou verificar se o Solver pode ser utilizado como ferramenta eficaz para a otimização da separação de pedidos da $O M X$. Para isso, como objetivos específicos procurou analisar o processo de recolhimento de pedidos na $O M X \mathrm{e}$ comparar o deslocamento em metros realizado sem o Solver com os resultados da utilização dele no recolhimento de materiais nas prateleiras.

Como consequência, poderá ser analisada a real necessidade da atual quantidade de pessoal envolvido no processo de separação de pedidos, ou seja, pode haver redução/remanejamento dos militares, servidores civis e/ou estivadores empregados na atividade de separação de pedidos.

Mesmo com uma diferença relativamente pequena em metros do executado atualmente e do proposto pelo Solver, cabe considerar que o universo de pesquisa pode ser majorado para outras OM que executam a mesma função do que a OM analisada, assim como a inclusão de diversos outros SJ.

No caso específico dos estivadores da $O M X$ a análise e a melhora do processo de separação de pedidos pode gerar uma economia financeira relevante, já que envolve um contrato licitatório que custa cerca de $\mathrm{R} \$ 2$ milhões por ano.

A OM objeto deste estudo foi escolhida por ser um dos Depósitos do Complexo Naval de Abastecimento com maior número mensal de Requisições de Material (RM) por OM clientes. Também, por contar com um dos maiores efetivos de pessoal envolvido na atividade de separação de pedidos. Ademais, por ser o Depósito com a maior diversidade de SJ sob responsabilidade de uma OMF.

Dessa forma, a $O M X$ contribuirá para o seu próprio reconhecimento como OMF moderna e tecnologicamente atualizada, contribuindo ainda mais para o aprimoramento do SAbM e estando alinhado às exigências da Administração Pública contemporânea. 
Além disso, este estudo buscou ser capaz de fornecer informações relevantes para apoiar decisões gerenciais e visou contribuir para a busca da excelência no cumprimento da missão da $\mathrm{MB}$ enquanto Administração Pública. A utilização de métodos quantitativos no apoio à tomada de decisões em cenários complexos ajuda a evitar o emprego de processos pouco criteriosos.

Por fim, como os dados deste trabalho referem-se somente a OM X, sugere-se replicar este estudo em outras OMF, considerando também outros SJ.

\section{REFERÊNCIAS BIBLIOGRÁFICAS}

ANGELINI, Julia et al. Utilização da Pesquisa Operacional para Otimização do Mix de Produtos em uma Empresa de Foz do Iguaçu, PR. Revista Pleiade. Jun-dez, 2017. Disponível em: <http://intranet.uniamerica.br/site/revista/index.php/pleiade/article/ download/352/309>. Acesso em: 13 ago. 2018.

BALLOU, R. H. Gerenciamento da cadeia de suprimentos: planejamento, organização e logística empresarial. $5^{\text {a }}$ ed. Porto Alegre: Bookman, 2006.

BRASIL. Constituição (1988). Constituição da República Federativa do Brasil. Diário Oficial [da] União. Brasília, DF, 05 out. 1988. Disponível em: $<$ http://www.tse.jus.br/legislacao/codigo-eleitoral/constituicao-federal/constituicao-darepublica-federativa-do-brasil >. Acesso em: 15 jul. 2018.

. Marinha do Brasil. $\boldsymbol{O M} \boldsymbol{X}$. Carta de Serviços. Rio de Janeiro, 2018.

. Marinha do Brasil. Secretaria-Geral da Marinha. SGM-107: normas gerais de administração. 6. rev. Brasília, 2015. Disponível em: <http://sgm.mb/PUB/Normas/SGM107-REV6.pdf>. Acesso em: 16 out. 2018. Acesso em: 22 ago. 2018.

Marinha do Brasil. Secretaria-Geral da Marinha. SGM-201: normas para a execução do abastecimento. 6.rev. Brasília, 2009. Disponível em: $<$ http://sgm.mb/PUB/Normas/SGM-201-REV6.pdf> Acesso em: 22 ago. 2018.

. Marinha do Brasil. Secretaria-Geral da Marinha. SGM-303: normas sobre gestão do material. 5. rev. Brasília, 2016. Disponível em: <http://sgm.mb/PUB/Normas/SGM-303REV5.pdf $>$ Acesso em: 23 ago. 2018.

CARDOSO, Andrea. Fundamentos da Pesquisa Operacional. Minas Gerais: UNIFAL, 2011. Disponível em: < https://www.coursehero.com/file/17419965/po/> Acesso em: 15 jul. 2018.

CUNHA, Claudio B.; SILVA, Marcos Roberto. A genetic algorithm for the problem of configuring a hub-and-spoke network for a LTL trucking company in Brazil. European Journal Of Operational Research, St. Louis, v. 1, n. 179, 2007. Disponível em: <

79 https://www.sciencedirect.com/science/article/abs/pii/S0377221705007344>. Acesso em: 23 mar. 2019.

GASNIER, D.; BANZATO, E. Armazém inteligente. Revista LOG, Movimentação e Armazenagem. São Paulo, n. 128. 2001. 
GIL, Antonio Carlos. Métodos e técnicas de pesquisa social. $6^{\text {a }}$. Ed. São Paulo: Atlas, 2008.

Hillier, Frederick S. Introduction to operations research/Frederick S. Hillier, Gerald J. Lieberman; cases developed by Karl Schmedders and Molly Stephens; tutorial software developed by Mark Hillier and Michael O’Sullivan.—7th ed, 2001. Disponível em $<$ https:// notendur.hi.is/kth93/3.20.pdf> Acesso em: 19 ago. 2019.

JUNIOR, Ilton CurtyDe et al. Estudo para Implementação de um Sistema de Roteirização e um Novo Centro de Distribuição para uma Empresa de Água Mineral do Sul de Minas Gerais. In: Simpósio de excelência em gestão e tecnologia. 2013, Rio de Janeiro. Anais... Rio de Janeiro: SEGET, 2013. Disponível em: . Acesso em: 23 mar. 2019.

LAPORTE, G.; GENDREAU, M.; POTVIN, J.Y.; SEMET, F. Classical and modern heuristics for the vehicle routing problem. International Transactions in Operational Research, v. 7, n. 4, 2000. Disponível em < http://www.scielo.br/scielo.php? script $=$ sci_nlinks\&ref $=000158 \&$ pid $=S 0104530 X 200300020000500015 \& \operatorname{lng}=p t>. \quad$ Acesso em: 23 mar. 2019.

LIMA, Isis Coelho et al. Otimização de rotas na gestão de resíduos: um estudo de caso na coleta de resíduos recicláveis do centro de ciências da saúde da UFRJ - $9^{\circ}$ Fórum Internacional de Resíduos Sólidos. Universidade de Brasília. Brasília. 2018. Disponível em: <http://www.institutoventuri.org.br/ojs/index.php/firs/article/view/813>. Acesso em: 17 set. 2018.

MARINS, Fernando Augusto Silva. Introdução à Pesquisa Operacional. Cultura Acadêmica: Universidade Estadual Paulista, Pró-Reitoria de Graduação, 2011. Disponível em: <https://www.cairu.br/biblioteca/arquivos/Ciencia/Introducao_pesquisa_operacional. pdf $>$. Acesso em: 17 set. 2018.

MARKONI, Marina de Andrade; LAKATOS, Eva Maria. Fundamentos da Metodologia Científica. 7a . ed. São Paulo: Atlas S.A., 2010.

MICROSOFT. Corporation. Definir e resolver um problema usando o Solver. Disponível em: $\quad<$ https://support.office.com/pt-br/article/definir-e-resolver-um-problema-usando-osolver-5d1a388f-079d-43ac-a7eb-f63e45925040>Acesso em: 18 jul. 2018

NETO, Aristides Fraga; CARDOSO, Patrícia Alcântara. Desenvolvimento de um sistema de roteirização de veículos em um armazém para apoio na decisão de coleta de produtos acabados. In: X Congresso Nacional de Excelência de Gestão, 8-9ago, 2014. Rio de Janeiro - RJ. Disponível em: $<$ http://www.inovarse.org/sites/default/files/T14_0162_8.pdf $>$ Acesso em: 18 jul. 2018

PRODANOV, Cleber Cristiano; FREITAS, Ernane Cesar de. Trabalho científico: métodos e técnicas da pesquisa e do trabalho acadêmico. 2. ed. Rio Grande do Sul: Feevale, 2013.

SOHN, Eugene. Simulated annealing algorithm for customer-centric location routing problem. Master of engineering in supply chain management at the Massachusetts Institute of Technology (MIT). Massachusetts, 2018.2018 Disponível em: $<$ https://dspace.mit.edu/handle/1721.1/117923> Acesso em: 18 set. 2018 
TORTOLA, Edgar Xavier; GALAN, Crislaine Rodrigues; CASTILHO, Ana Carolina Britto. Análise da Proposta da Melhoria de uma indústria de bebidas. Revista Uningá Reweiw. v. 31, n. 1, p. 06. jan, 2018. ISSN 2178-2571. Disponível em: $<$ http://revista.uninga.br/index.php/uningareviews/article/view/2047>Acesso em: 27 set. 2018

TREVISAN, Eduardo Carlos et al. Utilização do método do caminho ótimo para roteirizar a coleta seletiva de embalagens cartonadas na cidade de embu das artes. International Conference on Network Entreprise e Logistic Management. São Paulo - SP, 2018. Disponível em: <https://www.researchgate.net/publication/325746014 UTILIZACAO_DO

METODO DO CAMINHO OTIMO PARA ROTEIRIZAR A COLETA SELETIVA D E_EMBALAGENS_CARTONADAS_NA_CIDADE_DE_EMBU_DAS_ARTES-SP> Acesso em: 18 jul. 2018.

VERGARA, Sylvia Constant. Projetos e relatórios de pesquisa em administração. 15 . ed. São Paulo: Atlas S.A., 2014.

Yin, Robert k. Estudo de Caso: planejamentos e métodos. 4a. Ed. Porto Alegre: Bookman, 2010 . 